Archive for

Organic Chemistry
Arkivoc 2019, part vi, 0-0

to be inserted by editorial office

\title{
Synthesis, characterization, analgesic and anti-inflammatory activity of new pyrazole derivatives
}

\author{
Pullagura Priyadarsini, ${ }^{a}$ Vallabhaneni Madhavarao, ${ }^{a^{*}}$ and Bodepudi Sudheer Chowdary ${ }^{b}$ \\ ${ }^{a}$ Dept. of Chemistry, Bapatla Engineering College, Bapatla, Acharya Nagarjuna University, Guntur (District), \\ Andhra Pradesh-522 101, India \\ ${ }^{b}$ Dept. of Pharmacology, Bapatla College of Pharmacy, Jawaharlal Nehru Technical University-K, \\ Bapatla, A.P., India \\ E-mail: vmrgpm@gmail.com
}

Received 09-25-2019

Accepted 12-23-2019

Published on line $01-16-2020$

\section{Abstract}

The synthesis of nine new pyrazole derivatives was achieved from $\beta$-hydroxy enones and hydrazines by using a trace of piperidine in methanol at room temperature. The use of piperidine provides more yield and purity of products in lesser time when compared to the other reagents. All synthetic compounds were characterized by their physical properties, NMR and LC mass spectral data. The pyrazole derivatives were screened for their analgesic and anti-inflammatory activities in vivo. The derivatives exhibited moderate to significant activities in comparison to control, and most of the pyrazoles were competent with standard drugs (Pentazocine and Indomethacin).

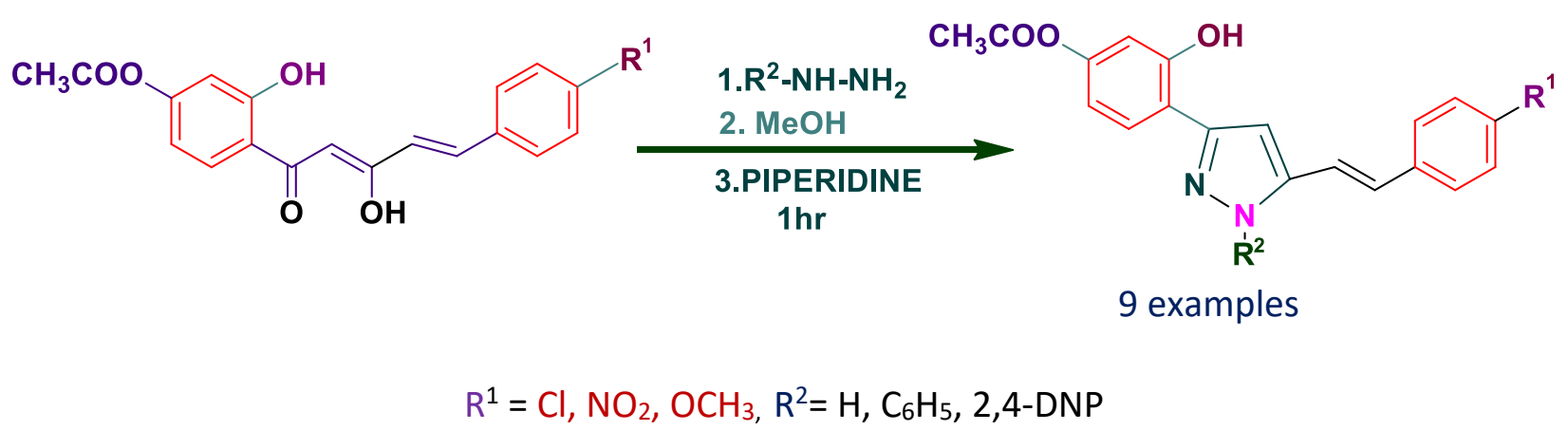

Keywords: Pyrazoles, $\beta$-hydroxy-enones, hydrazines, piperidine, anti-inflammatory activity, analgesic activity 


\section{Introduction}

Pyrazoles are important heteroaromatic compounds with two nitrogen atoms in the five-membered ring system. They play a vital role in the pharmaceutical ${ }^{1}$ and agrochemical industry. ${ }^{2,3}$ They are classed as azoles. ${ }^{4}$ Azoles occupy a domain of interest in natural and synthetic chemistry. Pyrazoles resemble benzene, and most of their properties were analyzed in comparison with the derivatives of benzene. ${ }^{5}$ Knorr synthesized triaryl pyrazoles for the first time, by condensation of hydrazines with $\beta$-diketones. ${ }^{6}$ Varma and co-workers developed a room temperature protocol for pyrazole synthesis using water as the solvent. ${ }^{7}$ The binding of heterocyclic compounds with an acyclic sugar moiety, forming thus acyclic-nucleosides, has commanded worldwide attention of many research groups because of their high potential to exhibit chemotherapeutic activity. ${ }^{8,9}$ Substituted pyrazole and its analogs have been used as precursors for the synthesis of various biologically active molecules.

The pyrazole nucleus is common in a number of biologically active molecules exhibiting antibacterial, ${ }^{10,11}$ antitubercular, ${ }^{12}$ anti-depressant, ${ }^{13}$ anti-inflammatory, ${ }^{14,15}$ analgesic, ${ }^{16}$ anticancer, ${ }^{17,18}$ antioxidant, ${ }^{19,20}$ and other activities. The efficiency of microwave heating in dramatically reducing reaction times has recently been proven in several fields of organic chemistry. ${ }^{21,22}$ There are numerous methods for synthesizing pyrazoles, ${ }^{23-25}$ among which is the 1,3-dipolar cycloaddition of diazo compounds (as 1,3-dipoles) with alkenes, ${ }^{23-26}$ or alkynes. ${ }^{27-29}$ The utility of diazo compounds in such reactions remains to be explored in new domino reactions, the tandem 1,3-dipolar cycloaddition / intramolecular - aza-addition/ oxidative aromatization, which has led to an efficient construction of substituted analogs of withasomnines from readily available acyclic starting materials with diazomethanes under mild conditions in a single step. ${ }^{30-32}$ There are still unexplored aspects of this area of research.

\section{Results and Discussion}

The importance and biological activities of pyrazoles led us to prepare new pyrazoles in three steps followed by a study of their analgesic and anti-inflammatory activities in vivo. The synthetic process initially involved condensation of cinnamic acids ${ }^{33}$ with $o$-hydroxy-4'-acetoxyacetophenones in pyridine using $\mathrm{POCl}_{3}$ as a condensing agent, to obtain 2'-cinnamoyloxyacetophenones (5-7). These were treated with powdered $\mathrm{KOH}$ in pyridine to give $\beta$-hydroxy-enones, ${ }^{34,35}$ and then converted into pyrazoles by treating with alkaline hydrazine. The procedure for the preparation of the desired pyrazoles 11-19 is given in Scheme 1.

The optimization of the reaction conditions was carried out with $\beta$-hydroxy-enones as model substrate using different bases and solvents, at room temperature (Table 1 ). The best results were attained when the reaction was run at room temperature with methanol as a solvent in the presence of piperidine in a catalytic amount, providing the new pyrazoles (11-19). Piperidine proved to be very effective in increasing the yields as well as the purity of the products within considerably less time than the other reagents. The completion of the reaction and purity of the products were monitored by thin-layer chromatography. Pinto et.al ${ }^{36}$ has reported some pyrazoles which were prepared from 2-styrylchromones under reflux conditions in methanol.

All the final products were confirmed from their spectral data, viz. ${ }^{1} \mathrm{H}$ and ${ }^{13} \mathrm{C}-\mathrm{NMR}$, LC-Mass. Signals in PMR at $\delta 6.54-7.15(1 \mathrm{H}, \mathrm{s})$ indicate $H(4)$ in pyrazole ring. The $\delta$ value at 7.27-7.90 was due to shielding of $\alpha$ protons in the styryl group, whereas $\beta$-protons resonate at relatively higher $\delta$ values because of polarization of $\pi$-electrons, in the range 7.40-7.93 ppm. The trans configuration of $\mathrm{H} \alpha$ and $\mathrm{H} \beta$ of styryl group was established 
from the corresponding coupling constant, $J_{H \alpha-H \beta} \approx 16 \mathrm{~Hz}$. The aromatic protons gave signals at various $\delta$ values depending on the nature and position of the substituent. Phenolic protons have appeared at $\delta$ 11.30-12.48 ppm. The methoxy protons gave a strong signal at 3.80 and methyl protons of the ester group at $\delta 2.08 \mathrm{ppm}$.

The ${ }^{13} \mathrm{C}-\mathrm{NMR}$ values obtained at $\delta 107,136$ and $147 \mathrm{ppm}$, confirmed the presence of the pyrazole group. The high $\delta$ values at 160,154 , etc. confirmed that the aromatic carbons substituted with $-\mathrm{OH},-\mathrm{OCH}_{3}$ groups, the values at $\delta 167-168 \mathrm{ppm}$ reveals the presence of carbonyl group in methyl ester.

All the synthesized final products showed strong peaks in their LC-Mass spectra at respective mass values in positive ion and negative ion modes.

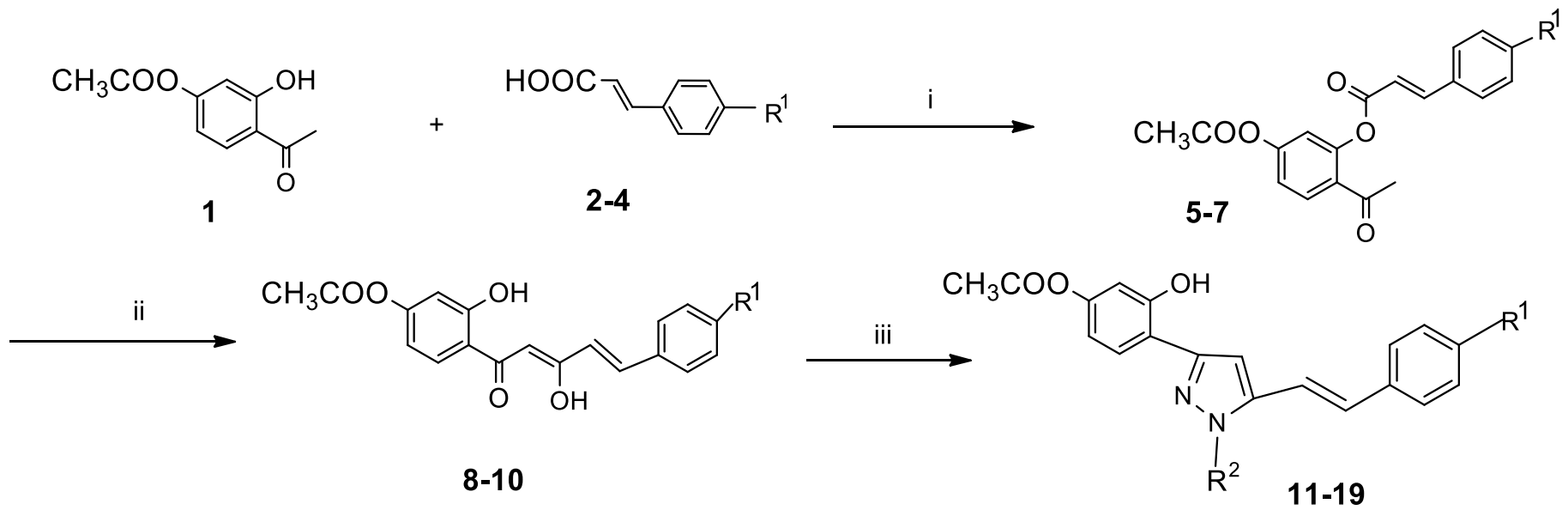
11. $\mathrm{R}^{1}=\mathrm{NO}_{2}, \mathrm{R}^{2}=2,4-\mathrm{DNP}$
14. $\mathrm{R}^{1}=\mathrm{Cl}, \mathrm{R}^{2}=2,4-\mathrm{DNP}$
17. $\mathrm{R}^{1}=\mathrm{OCH}_{3}, \mathrm{R}^{2}=2,4-\mathrm{DNP}$
12. $\mathrm{R}^{1}=\mathrm{NO}_{2}, \mathrm{R}^{2}=\mathrm{Ph}$
15. $\mathrm{R}^{1}=\mathrm{Cl}, \mathrm{R}^{2}=\mathrm{Ph}$
18. $R^{1}=\mathrm{OCH}_{3}, \mathrm{R}^{2}=\mathrm{Ph}$
13. $R^{1}=N_{2}, R^{2}=H$
16. $\mathrm{R}^{1}=\mathrm{Cl}, \mathrm{R}^{2}=\mathrm{H}$
19. $R^{1}=\mathrm{OCH}_{3} \cdot \mathrm{R}^{2}=\mathrm{H}$

Scheme 1. Reagents and Conditions: (i) $\mathrm{POCl}_{3}$, pyridine; rt. 4 hrs; (ii) $\mathrm{KOH}$, dry pyridine, , rt. 1-2 hrs; (iii) $\mathrm{R}^{2}-\mathrm{NH}-$ $\mathrm{NH}_{2}, \mathrm{MeOH}$, piperidine, $1 \mathrm{hr}$ stirring at rt.

Table 1. Optimization table

\begin{tabular}{clcc}
\hline Entry & \multicolumn{1}{c}{ Solvent } & $\begin{array}{c}\text { Time } \\
\text { (Min.) }\end{array}$ & Yield \% \\
\hline 1 & $\mathrm{DCM}+\mathrm{NaOH}$ & $80-90$ & $80 \%$ \\
2 & $\mathrm{MeOH}$ & $90-120$ & $75 \%$ \\
3 & $\mathrm{MeOH}+$ piperidine & $50-60$ & $90 \%$ \\
4 & $\mathrm{MeOH}+$ triethylamine & $80-90$ & $70 \%$ \\
\hline
\end{tabular}


Table 2. Substrate scope for synthesis of substituted pyrazoles

\begin{tabular}{|c|c|c|c|}
\hline & Compound name & Yield (\%) & M.P \\
\hline 1 & $\begin{array}{l}\text { 3-(4-Acetoxy-2-hydroxyphenyl)-5-(4-nitrostyryl)-1- } \\
\text { (2,4-dinitrophenyl)pyrazole (11) }\end{array}$ & $0.65 \mathrm{~g}(91.5 \%)$ & $219-220^{\circ} \mathrm{C}$ \\
\hline 2 & $\begin{array}{l}\text { 3-(4-Acetoxy-2-hydroxyphenyl)-5-(4-nitrostyryl)-1- } \\
\text { phenylpyrazole (12) }\end{array}$ & 0.550 g. (93\%) & $231-232^{\circ} \mathrm{C}$ \\
\hline 3 & $\begin{array}{l}\text { 3-(4-Acetoxy-2-hydroxyphenyl)-5-(4-nitrostyryl)- } \\
\text { pyrazole (13) }\end{array}$ & $0.360 \mathrm{~g}(91.1 \%)$ & $246-247^{\circ} \mathrm{C}$ \\
\hline 4 & $\begin{array}{l}\text { 3-(4-Acetoxy-2-hydroxyphenyl)-5-(4-chlorostyryl)- } \\
\text { 1-(2,4-dinitrophenyl)pyrazole (14) }\end{array}$ & 0.300 g. $(83 \%)$ & $169-170^{\circ} \mathrm{C}$ \\
\hline 5 & $\begin{array}{l}\text { 3-(4-Acetoxy-2-hydroxyphenyl)-5-(4-chlorostyryl)- } \\
\text { 1-phenylpyrazole (15) }\end{array}$ & 0.320 g. (88\%) & $89-90^{\circ} \mathrm{C}$ \\
\hline 6 & $\begin{array}{l}\text { 3-(4-Acetoxy-2-hydroxyphenyl)-5-(4-chlorostyryl)- } \\
\text { pyrazole (16) }\end{array}$ & 0.260 g. (86.6\%) & $223-224^{\circ} \mathrm{C}$ \\
\hline 7 & $\begin{array}{l}\text { 3-(4-Acetoxy-2-hydroxyphenyl)-5-(4-methoxy- } \\
\text { styryl-1-(2,4-dinitrophenyl)pyrazole (17) }\end{array}$ & 0.340 g. (94.4\%) & $186-187^{\circ} \mathrm{C}$ \\
\hline 8 & $\begin{array}{l}\text { 3-(4-Acetoxy-2-hydroxyphenyl)-5-(4-methoxy- } \\
\text { styryl)-1-phenylpyrazole (18) }\end{array}$ & 0.350 g. $(81 \%)$ & $226-227^{\circ} \mathrm{C}$ \\
\hline 9 & $\begin{array}{l}\text { 3-(4-Acetoxy-2-hydroxyphenyl)-5-(4-methoxy- } \\
\text { styryl)pyrazole (19) }\end{array}$ & 0.230 g. $(76.6 \%)$ & $124-125^{\circ} \mathrm{C}$ \\
\hline
\end{tabular}

\section{Biological testing data}

All pyrazole derivatives except 12 exhibited thermal pain response much more effectively than Pentazocine in the same dose (Figure 1). This could be due to the pyrazole ring structure with the nature and position of substituents in the styryl group and at N(1) of pyrazole. All the compounds tested by Eddy's hot plate method showed better activity at the dosage of $30 \mathrm{mg} / \mathrm{kg}$. The significant activity of all pyrazoles was due to the $-\mathrm{Cl},-$ OMe at position 4 of the styryl group. In the absence of these groups, the compounds showed lower or nonsignificant activity, especially compound 12 has much lesser activity at 60 minutes, while 11, 13, and 15 have lesser activity at 30 minutes.

The synthesized pyrazoles exhibited significant anti-inflammatory activity when compared to the control group (Figure 2). The anti-inflammatory activities of compounds 11-19 were studied in the carrageenaninduced edema model of inflammation in mice. All the pyrazole derivatives showed significant antiinflammatory activity. The compound 16, 17, showed better activity might be due to the presence of methoxyl and chloro substituents at 4-position in the styryl ring of pyrazole. The compounds $\mathbf{1 1}$ and $\mathbf{1 2}$ exhibited good activity might well be due to the presence of the nitro group in the styryl ring. Hence, the 
anti-inflammatory activity of pyrazole derivatives was increased with the styryl group substituted by an electron releasing group at position 4.

Tables S1 and S2 (Supplementary file) give full details of the analgesic and anti-inflammatory testing data of the individual compounds reported here. The results are summarized in Figures 1 and 2 below.

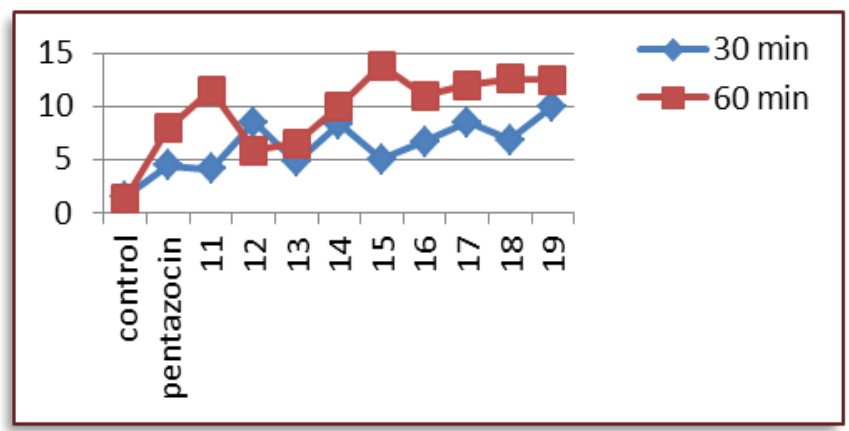

Figure 1. Graph for Analgesic activity.

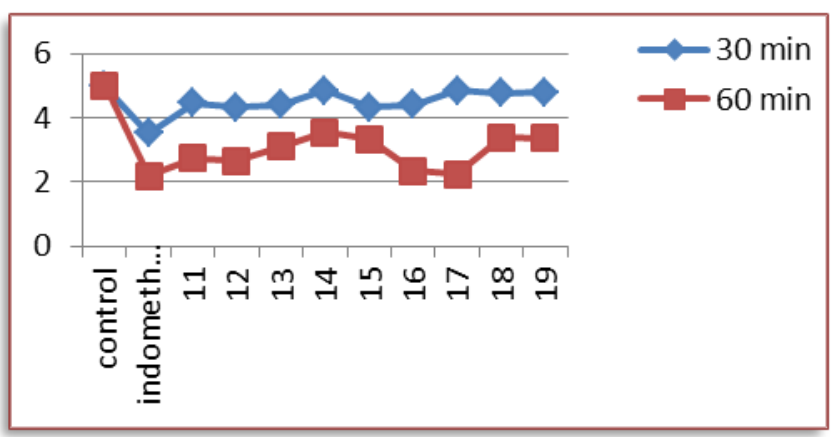

Figure 2. Graph for Anti-inflammatory activity.

\section{Conclusions}

We have synthesized new pyrazole derivatives containing styryl groups at one position of pyrazole ring. The yield and purity of the final products were enhanced by using a trace amount of piperidine at the third stage of preparation. All reactions gave their target products in good to excellent yield. The reactions were rapid, facile and accomplished at room temperature. Almost all the synthesized compounds exhibited significant analgesic and anti-inflammatory activity against Pentazocine and Indomethacin in vivo of albino mice and rats respectively

\section{Experimental Section}

General: Melting points were measured by a Stuart Scientific melting point apparatus in open capillaries. NMR spectra were recorded on a Bruker spectrometer operating at $400 \mathrm{MHz}$ for proton \& $100 \mathrm{MHz}$ for ${ }^{13} \mathrm{C}$-spectra, using DMSO- $d_{6}$ as a solvent with TMS as an internal standard. Mass spectra were recorded on an Agilent-1100 period LC-MSD. Elemental analysis was performed using a EURO EA 3000 instrument. Acme silica gel-G and Merck silica gel (100 to 200, 60 to 120 meshes) were used for analytical TLC and column chromatography respectively. All other analytical grade chemicals and solvents were obtained from commercial sources and used as received.

The three ester compounds (5-7) were obtained by treating substituted 2-hydroxy-4-acetoxyacetophenones (1 $\mathrm{mmol})$ with cinnamic acids $(1.2 \mathrm{mmol})$ in pyridine solution $(17.8 \mathrm{mmol})$ in the presence of phosphorus oxychloride $(3.0 \mathrm{mmol})$ as condensing agent according to the literature procedure. ${ }^{34,35}$ All esters were converted into the respective $\beta$-hydroxy-enones (8-10) by stirring a pyridine $(4.3 \mathrm{ml})$ solution of the ester (1.2 $\mathrm{mmol}$ ) containing $250 \mathrm{mg}$ of powdered potassium hydroxide for 2 hours following the method reported in the literature. ${ }^{34,35}$ 
The obtained $\beta$-hydroxy-enone (1mmol) and hydrazine $(1 \mathrm{mmol})$, were dissolved in $\mathrm{MeOH}(5 \mathrm{~mL})$, and the resulting solution was stirred for about one hour at room temperature in the presence of a catalytic amount of piperidine. The completion of the reaction was monitored by TLC, and the reaction mixture was poured into ice-cold water containing $20 \% \mathrm{HCl}$. The solid thus obtained was filtered, washed, dried and purified by column chromatography over silica gel using hexane: EtOAc (4.75:0.25) as eluent. The resulting substituted pyrazole was recrystallized from a mixture of ethyl acetate and hexane.

3-(4-Acetoxy-2-hydroxyphenyl)-5-(4-nitrostyryl)-1-(2,4-dinitrophenyl)pyrazole (11): Following the general procedure, 11 was obtained from 1-(4-acetoxy-2-hydroxyphenyl)-5-(4-nitrophenyl)-3-hydroxypenta-2,4-dien1-one (1 mmol, 0.5g,) and 2,4-dinitrophenylhydrazine (1mmol 0.198g), as a brown solid. Yield 0.65g (91.5\%), mp 219-220 ${ }^{\circ} \mathrm{C} .{ }^{1} \mathrm{H}-\mathrm{NMR}$ (DMSO-d $\left.d_{6}\right): \delta 11.35(1 \mathrm{H}, \mathrm{br}, \mathrm{s}, \mathrm{Ar}-\mathrm{OH}), 8.88(1 \mathrm{H} \mathrm{s}), 8.8(1 \mathrm{H}, \mathrm{s}) 7.77-7.71(3 \mathrm{H}, \mathrm{d}),, 8.369-$ 8.352(3H,d ),7.46 (1H, d, J = $16.0 \mathrm{~Hz}, \mathrm{H}-\alpha), 7.48(1 \mathrm{H}, \mathrm{d}, J=16.0 \mathrm{~Hz}, \mathrm{H}-\beta), 7.07(1 \mathrm{H}, \mathrm{s}$, pyrazole), $7.106(\mathrm{IH}, \mathrm{d})$, $6.85(1 \mathrm{H}, \mathrm{s}), 2.087(\mathrm{~s}, 3 \mathrm{H} \mathrm{Me}) .{ }^{13} \mathrm{C}$ NMR (DMSO-d $): \delta 167.34$ (C=O), 157.55, 151.39, 147.09, 144.71, 144.65, $136.69,130.07,129.74,128.88,124.29,123.05,120.5,118.47,116.2,115.94,107.5,100.6,20.8(M e)$. LC-MS (ESI, negative ion mode): $m / z 530(\mathrm{M}-\mathrm{H})^{-}$. Anal. Calcd. (\%) For $\mathrm{C}_{25} \mathrm{H}_{17} \mathrm{~N}_{5} \mathrm{O}$ : $\mathrm{C}, 56.49 ; \mathrm{H}, 3.20 ; \mathrm{N}, 13.18$. Found (\%): C, 56.51; H, 3.27; N, 13.17\%.

3-(4-Acetoxy-2-hydroxy phenyl)-5-(4-nitrostyryl)-1-phenylpyrazole (12): Following the general procedure, 12 was obtained from 1-(4-acetoxy-2-hydroxyphenyl)-5-(4-nitrophenyl)-3-hydroxypenta-2,4-dien-1-one (1 mmol, $0.5 \mathrm{~g})$ and phenylhydrazine $(1 \mathrm{mmol}, 0.144 \mathrm{~g})$, as light brown solid. Yield: $0.550 \mathrm{~g},(93 \%), \mathrm{mp} 231-232{ }^{\circ} \mathrm{C} .{ }^{1} \mathrm{H}-$ NMR (DMSO-d $\left.)_{6}\right): \delta 12.717(1 \mathrm{H}, \mathrm{br}, \mathrm{s}, \mathrm{Ar} \mathrm{OH}), 8.07(2 \mathrm{H}, \mathrm{d}), 7.919(1 \mathrm{H}, \mathrm{d}, J=16.0 \mathrm{~Hz}, \mathrm{H}-\alpha), 7.935(1 \mathrm{H}, \mathrm{d}, J=16.0$ $\mathrm{Hz}, \mathrm{H}-\beta), 7.6-7.9(5 \mathrm{H}, \mathrm{m}), 7.7(2 \mathrm{H}, \mathrm{d}), 7.45(1 \mathrm{H}, \mathrm{d}), 6.98(1 \mathrm{H}, \mathrm{d}) 6.904(1 \mathrm{H}-\mathrm{s}$, pyrazole) $6.79(1 \mathrm{H}, \mathrm{s}) 2.087(3 \mathrm{H}, \mathrm{S})$. ${ }^{13} \mathrm{C}$ NMR (DMSO- $\left.d_{6}\right): \delta 167.34(\mathrm{C}=0), 157.55,151.39,147.09,144.71,140.5,136.69,130.07,129.74,129.21$, $128.88,124.75,124.15,123.05,120.5,116.2,115.94,107.5,100.6,20.8$ (Me). LC-MS (ESI, negative ion mode): $\mathrm{m} / \mathrm{z} 440(\mathrm{M}-\mathrm{H})^{-}$. Anal. Calcd. (\%) for $\mathrm{C}_{25} \mathrm{H}_{19} \mathrm{~N}_{3} \mathrm{O}_{5}$ : C, 68.03; H, 4.31; N, 9.52. Found (\%): C, 68.13; H, 4.57; N, 9.48 .

3-(4-Acetoxy-2-hydroxyphenyl)-5-(4-nitrostyryl)pyrazole (13): Following the general procedure, 13 was obtained from 1-(4-acetoxy-2-hydroxyphenyl)-5-(4-nitrophenyl)-3-hydroxypenta-2,4-dien-1-one (0.33g), hydrazine sulfate $(1 \mathrm{mmol}, 0.130 \mathrm{~g})$, as light brownish colour solid. Yield-0.360g, (91.1\%), mp $246-247^{0} \mathrm{C} .{ }^{1} \mathrm{H}-$ NMR (DMSO-d $): \delta 14.23(\mathrm{NH}, 1 \mathrm{H}, \mathrm{s}), 12.717(1 \mathrm{H}, \mathrm{br}, \mathrm{s}, \operatorname{Ar}-\mathrm{OH}), 8.301(2 \mathrm{H}, \mathrm{d}), 7.77(1 \mathrm{H}, \mathrm{d}, J=16.0 \mathrm{~Hz}, \mathrm{H}-\alpha)$, $7.845(1 \mathrm{H}, \mathrm{d}, \mathrm{J}=16.0 \mathrm{~Hz}, \mathrm{H}-\beta), 7.885(1 \mathrm{H}, \mathrm{d}), 7.7(2 \mathrm{H}, \mathrm{d}), 7.116(1 \mathrm{H}, \mathrm{d}), 6.8(1 \mathrm{H}, \mathrm{s}), 6.7(1 \mathrm{H}, \mathrm{s}$, pyrazole $), 2.087$ $(3 \mathrm{H}, \mathrm{s}) .{ }^{13} \mathrm{C}-\mathrm{NMR}\left(\mathrm{DMSO}-d_{6}\right): \delta 167.34(\mathrm{C}=\mathrm{O}), 157.55,151.39,147.09,144.71,136.69,130.07,129.74,128.88$, 124.15, 123.05, 120.5, 116.2, 115.94, 107.5, 100.6, 20.8 (Me). LC-MS (ESI, positive ion mode): $\mathrm{m} / z 366(\mathrm{M}+\mathrm{H})^{+}$. Anal. Calcd. (\%) for $\mathrm{C}_{19} \mathrm{H}_{15} \mathrm{~N}_{3} \mathrm{O}_{5}$ : $\mathrm{C}, 62.4 ; \mathrm{H}, 4.1 ; \mathrm{N}, 11.5$. Found (\%): C, 62.39; $\mathrm{H}, 4.12 ; \mathrm{N}, 11.48$.

3-(4-Acetoxy-2-hydroxyphenyl)-5-(4-chlorostyryl)-1-(2,4-dinitrophenyl)pyrazole (14): Following the general procedure, 14 was obtained from 1-(4-acetoxy-2-hydroxyphenyl)-5-(4-chlorophenyl)-3-hydroxypenta-2,4-dien1-one (1 mmol, 0.25g) and 2,4-dinitrophenylhydrazine (1mmol, $0.198 \mathrm{~g})$, as a brownish orange solid. Yield 300 mg (83\%), mp 169- $170{ }^{\circ} \mathrm{C} .{ }^{1} \mathrm{H}-\mathrm{NMR}$ (DMSO- $\left.d_{6}\right): \delta 12.4(1 \mathrm{H}, \mathrm{br}, \mathrm{s}, \mathrm{Ar}-\mathrm{OH}), 8.8(1 \mathrm{H}, \mathrm{s}), 8.6(1 \mathrm{H}, \mathrm{d}), 8.0(1 \mathrm{H}$, d), $7.8(1 \mathrm{H}, \mathrm{d}), 7.7(2 \mathrm{H}, \mathrm{d}), 7.56(1 \mathrm{H}, \mathrm{d}, J=16.0 \mathrm{~Hz}, \mathrm{H}-\alpha), 7.6(1 \mathrm{H}, \mathrm{d}, J=16.0 \mathrm{~Hz}, \mathrm{H}-\beta), 7.4(2 \mathrm{H}, \mathrm{d}), 7.17(1 \mathrm{H}, \mathrm{d}), 7.15$ $\left(1 \mathrm{H}, \mathrm{s}\right.$, pyrazole), $6.8(1 \mathrm{H}, \mathrm{s}), 2.09(3 \mathrm{H}, \mathrm{s}) .{ }^{13} \mathrm{C}-\mathrm{NMR}$ (DMSO- $\left.d_{6}\right): \delta 167.34(\mathrm{C}=0), 157.55,151.39,147.07,144.65$, 136.6, 134.65 (Cl), 133.2, 129.2, 129.8, 128.8, 126.6, 126.3, 122.7, 120.5, 118.47, 115.94, 107.5, 103.4, 100.6, 20.8 (Me). LC-MS (ESI, positive ion mode): $\mathrm{m} / \mathrm{z} 521.5(\mathrm{M}+\mathrm{H})^{+}$. Anal. Calcd.(\%) for $\mathrm{C}_{25} \mathrm{H}_{17} \mathrm{ClN}_{4} \mathrm{O}_{7}: \mathrm{C}, 57.64 ; \mathrm{H}$, 3.27; $\mathrm{N}, 10.7 ; \mathrm{Cl}, 6.82$. Found (\%): C, 57.84; H, 3.25; N, 10.4 . 
3-(4-Acetoxy-2-hydroxyphenyl)-5-(4-chlorostyryl)-1-phenylpyrazole (15): Following the general procedure, 15 was obtained from 1-(4-acetoxy-2-hydroxyphenyl)-5-(4-chlorophenyl)-3-hydroxypenta-2,4-dien-1-one (1 $\mathrm{mmol}, 0.3 \mathrm{~g}$ ) and phenylhydrazine $(1 \mathrm{mmol}, 0.144 \mathrm{~g})$, as snuff-colored solid. Yield $320 \mathrm{mg},(88 \%), \mathrm{mp} 89-90^{\circ} \mathrm{C}$. ${ }^{1} \mathrm{H}-N M R\left(D M S O-d_{6}\right): \delta 12.39(1 \mathrm{H}, \mathrm{br}, \mathrm{s}, \mathrm{Ar}-\mathrm{OH}), 7.93-7.89(5 \mathrm{H}, \mathrm{m}), 7.7(2 \mathrm{H}, \mathrm{d}), 7.1-7.2(2 \mathrm{H}, \mathrm{d}),, 7.4(2 \mathrm{H}, \mathrm{d}), 7.55$ $(1 \mathrm{H}, \mathrm{d}, J=6.0 \mathrm{~Hz}, \mathrm{H}-\alpha), 7.594(1 \mathrm{H}, \mathrm{d}, J=16.0 \mathrm{~Hz}, \mathrm{H}-\beta), 6.785(1 \mathrm{H}, \mathrm{s}$, pyrazole), $6.8(1 \mathrm{H}, \mathrm{s}), 2.19(3 \mathrm{H}, \mathrm{s}, \mathrm{Me})$. ${ }^{13}$ CNMR (DMSO- $\left.d_{6}\right): \delta 167.3(C=0), 158.8,153.2,147.07,142.3,137.5,134.65(\mathrm{Cl}), 133.2,129.8,129.2,128.8$, 126.3, 124.7, 122.7, 120.5, 114.6, 107.1, 100.6, 20.8 (Me). LC-MS (ESI, positive ion mode): $m / z 431.5(M+H){ }^{+}$. Anal. Calcd. (\%) for $\mathrm{C}_{25} \mathrm{H}_{19} \mathrm{ClN}_{2} \mathrm{O}_{3}$ : C, 69.69; $\mathrm{H}, 4.41 ; \mathrm{N}, 6.50$. Found (\%): C, 69.78; $\mathrm{H}, 4.70 ; \mathrm{N}, 6.48 \%$.

3-(4-Acetoxy-2-hydroxyphenyl)-5-(4-chlorostyryl)pyrazole (16): Following the general procedure, 16 was obtained from 1-(4-acetoxy-2-hydroxyphenyl)-5-(4-chlorophenyl)-3-hydroxypenta-2,4-dien-1-one (1mmol, 0.3 gm), hydrazine sulfate ( $1 \mathrm{mmol}, 0.13 \mathrm{~g})$, as light brownish solid. Yield $260 \mathrm{mg}(86.6 \%), \mathrm{mp} 223-224{ }^{\circ} \mathrm{C} .{ }^{1} \mathrm{H}-\mathrm{NMR}$ (DMSO-d $\left.)_{6}\right): \delta 14.23(\mathrm{NH}, 1 \mathrm{H}, \mathrm{s}), 12.4(1 \mathrm{H}, \mathrm{br}, \mathrm{s}, \mathrm{Ar}-\mathrm{OH}), 7.3(1 \mathrm{H}, \mathrm{d}), 7.7(2 \mathrm{H}, \mathrm{d}), 7.4(2 \mathrm{H}, \mathrm{d}), 7.27(1 \mathrm{H}, \mathrm{d}, J=16.0$ $\mathrm{Hz}, \mathrm{H}-\alpha), 7.63(1 \mathrm{H}, \mathrm{d}, J=16.0 \mathrm{~Hz}, \mathrm{H}-\beta), 7.1(1 \mathrm{H}, \mathrm{d}), 6.8(1 \mathrm{H}, \mathrm{s}), 6.54\left(1 \mathrm{H}, \mathrm{s}\right.$, pyrazole), $2.09(3 \mathrm{H}, \mathrm{s}) .{ }^{13} \mathrm{C}-\mathrm{NMR}$ (DMSO-d $)_{6}$ : $\delta 167.34$ (C=O), 157.55, 151.39, 147.09, 136.6, 134.65 (Cl), 133.2, 129.8, 129.21, 128.8, 126.3, 122.7, 120.5, 115.94, 107.5, 100.6, 20.8 (Me). LC-MS (ESI, negative ion mode): $\mathrm{m} / \mathrm{z}-353.5$ (M-H)-. Anal. Calcd (\%). for $\mathrm{C}_{19} \mathrm{H}_{15} \mathrm{ClN}_{2} \mathrm{O}_{3}$ : C, 64.3; $\mathrm{H}, 4.2 ; \mathrm{N}, 7.8$. Found (\%): $\mathrm{C}, 64.4 ; \mathrm{H}, 4.25 ; \mathrm{N}, 7.48$.

3-(4-Acetoxy-2-hydroxyphenyl)-5-(4-methoxystyryl-1-(2,4-dinitrophenyl)pyrazole (17): Following the general 17 was obtained from 1-(4-acetoxy-2-hydroxyphenyl)-5-(4-methoxyphenyl)-3-hydroxypenta-2,4-dien-1-one (1 mmol, $0.3 \mathrm{gm})$ and 2,4-dinitrophenylhydrazine $(1 \mathrm{mmol}, 0.198 \mathrm{~g})$, as brownish orange solid. Yield $340 \mathrm{mg}$ (94.4\%), mp 186-187 ${ }^{\circ} \mathrm{C} .{ }^{1} \mathrm{H}-\mathrm{NMR}$ (DMSO-d $)$ : $\delta 11.35(1 \mathrm{H}, \mathrm{br}, \mathrm{s}, \mathrm{Ar}-\mathrm{OH}), 8.87(1 \mathrm{H}, \mathrm{s}), 8.84(1 \mathrm{H}, \mathrm{d}), 7.8(2 \mathrm{H}, \mathrm{d})$, $7.3(1 \mathrm{H}, \mathrm{d}, J=16.0 \mathrm{~Hz}, \mathrm{H}-\alpha), 7.40(1 \mathrm{H}, \mathrm{d}, J=16.0 \mathrm{~Hz}, \mathrm{H}-\beta), 7.2(2 \mathrm{H}, \mathrm{d}), 7.14(1 \mathrm{H}, \mathrm{d}), 7.0(1 \mathrm{H}, \mathrm{s}$, pyrazole) $6.9(2 \mathrm{H}$, d), $6.8(1 \mathrm{H}, \mathrm{s}), 3.8\left(3 \mathrm{H}, \mathrm{s}, \mathrm{OCH}_{3}\right), 2.08(3 \mathrm{H}, \mathrm{s}) .{ }^{13} \mathrm{C}-\mathrm{NMR}$ (DMSO- $\left.d_{6}\right): \delta 167.34(\mathrm{C}=\mathrm{O}), 160.4,157.5,151.3,147.07$, 144.6, 136.6, 133.2, 128.9, 114.3, 128.8, 126.6, 126.3, 122.7, 120.5, 118.4, 115.9, 107.5, 103.4, 100.6, 55.5 $\left(\mathrm{OCH}_{3}\right) 20.8$ (Me). LC-MS (ESI, Negative ion mode): $\mathrm{m} / \mathrm{z} 515(\mathrm{M}-\mathrm{H})^{-}$. Anal. Calcd.(\%) for $\mathrm{C}_{26} \mathrm{H}_{20} \mathrm{~N}_{4} \mathrm{O}_{8}: \mathrm{C}, 60.46 ; \mathrm{H}$, 3.87 ; N, 10.85. Found: C, 60.49; H, 3.39; N, 10.83\%.

3-(4-Acetoxy-2-hydroxyphenyl)-5-(4-methoxystyryl)-1-phenylpyrazole (18): Following the general procedure 18 was obtained from 1-(4-acetoxy-2-hydroxyphenyl)-5-(4-methoxyphenyl)-3-hydroxypenta-2,4- dien-1-one (1 mmol, $0.3 \mathrm{~g}$ ) and phenylhydrazine (1 mmol, 0.144gm), as brownish yellow solid. Yield $350 \mathrm{mg},(81 \%), \mathrm{mp} 226-$ $227{ }^{\circ} \mathrm{C} .{ }^{1} \mathrm{H}-N M R\left(D M S O-d_{6}\right): \delta 11.35(1 \mathrm{H}, \mathrm{br}, \mathrm{s}, \mathrm{Ar}-\mathrm{OH}), 7.5-7.8(5 \mathrm{H}, \mathrm{m}), 7.3(1 \mathrm{H}, \mathrm{d}, J=16.0 \mathrm{~Hz}, \mathrm{H}-\alpha), 7.4(1 \mathrm{H}, \mathrm{d}, J$ $=16.0 \mathrm{~Hz}, \mathrm{H}-\beta), 7.38(1 \mathrm{H}, \mathrm{s}), 7.2(2 \mathrm{H}, \mathrm{d}), 7.1(1 \mathrm{H}, \mathrm{d}), 6.7(1 \mathrm{H}, \mathrm{s}$, pyrazole) $6.9(2 \mathrm{H}, \mathrm{d}), 6.8(1 \mathrm{H} \mathrm{d}), 3.8(3 \mathrm{H}, \mathrm{s}$, $\mathrm{OCH} 3$ ), $2.08(3 \mathrm{H}, \mathrm{s}) .{ }^{13} \mathrm{C}-\mathrm{NMR}$ (DMSO-d $)$ : $\delta 167.34$ (C=O), 160.4, 157.5, 151.3, 147.07, 142.3(C-N), 136.69, 133.2, 129.2, 128.9, 128.8, 127.5, 126.3, 124.7, 122.7, 120.5, 115.9, 114.3, 107.5, 100.6, 55.5(OCH $\mathrm{OCH}_{3} 20.8(\mathrm{Me})$. LC-MS (ESI, positive ion mode): $m / z 427(\mathrm{M}+\mathrm{H})^{+}$.Anal. Calcd. (\%) For $\mathrm{C}_{26} \mathrm{H}_{22} \mathrm{~N}_{2} \mathrm{O}_{4}$ : C, 73.24; $\mathrm{H}, 5.16 ; \mathrm{N}, 6.57$. Found: C, 73.30; $\mathrm{H}, 5.17 ; \mathrm{N}, 6.48$.

3-(4-Aceoxy-2-hydroxyphenyl)-5-(4-methoxystyryl)pyrazole (19): Following the general procedure, 19 was obtained from 1-(4-acetoxy-2-hydroxyphenyl)-5-(4-methoxyphenyl)-3-hydroxypenta-2,4-dien-1-one (1 mmol, $0.3 \mathrm{gm})$, hydrazine sulfate $(1 \mathrm{mmol}, 0.13 \mathrm{gm})$ as light brownish solid. Yield $230 \mathrm{mg},(76.6 \%), \mathrm{mp} 124-125^{\circ} \mathrm{C} .{ }^{1} \mathrm{H}-$ NMR (DMSO-d $\left.d_{6}\right): \delta 11.35(1 \mathrm{H}, \mathrm{br}, \mathrm{s}, \mathrm{Ar}-\mathrm{OH}), 7.5-7.8(5 \mathrm{H}, \mathrm{m}), 7.3(1 \mathrm{H}, \mathrm{d}, J=16.0 \mathrm{~Hz}, \mathrm{H}-\alpha), 7.4(1 \mathrm{H}, \mathrm{d}, J=16.0 \mathrm{~Hz}$, $\mathrm{H}-\beta), 7.38(1 \mathrm{H}, \mathrm{s}), 7.2(2 \mathrm{H}, \mathrm{d}), 7.1(1 \mathrm{H}, \mathrm{d}), 6.7(1 \mathrm{H}, \mathrm{s}$, pyrazole) $6.9(2 \mathrm{H}, \mathrm{d}), 6.8(1 \mathrm{H} \mathrm{d}), 3.8(3 \mathrm{H}, \mathrm{s}, \mathrm{OCH} 3), 2.08(3 \mathrm{H}$, s). ${ }^{13} \mathrm{C}-N M R\left(D M S O-d_{6}\right): \delta 167.3(C O), 160.4,157.5,151.3,147.07,136.69,133.2,128.9,128.8,126.3,122.7$, $120.5,114.3,115.9,107.5,100.6,55.5\left(\mathrm{OCH}_{3}\right), 20.8(\mathrm{Me})$. LC-MS (ESI, positive ion mode): $\mathrm{m} / \mathrm{z} 351(\mathrm{M}+\mathrm{H})^{+}$. Anal. Calcd. (\%) For $\mathrm{C}_{20} \mathrm{H}_{18} \mathrm{~N}_{2} \mathrm{O}_{4}$ : C, 68.57; $\mathrm{H}, 5.14 ; \mathrm{N}, 8$. Found (\%): C, 68.30; $\mathrm{H}, 5.15 ; \mathrm{N}, 7.98$. 


\section{Biological activity}

\section{Analgesic activity (Eddy's hot plate method)}

The activity was studied by Eddy's hot plate method $^{37}$ in albino mice. The hot plate consists of an electrically heated surface with a controlled temperature of $55 \pm 1^{\circ} \mathrm{C}$. Male albino mice of either sex with weight of about 26.5-47 gm. were placed on a hot plate and time of licking or jumping is recorded by stopwatch, before drug administration. The latency time is recorded after $0,30,60,120$ minutes following oral or subcutaneous administration of the test compound.

Male albino mice were selected and divided into eleven groups, containing three animals in each group. Weight matched animals were used in each group. These animals were fasted for twenty-four hours before the experiment. Animals of Group-I considered as control, were administered with 1\% Acacia suspension. Animals of Group-II were treated with Pentazocine (standard drug, $30 \mathrm{mg} / \mathrm{kg}$ ), which was considered as standard group. Animals of Group- III-XI were administered with the test sample and treated with different concentrations. The reaction time for each mouse was recorded at the time interval of 0, 30, 60 and 120 min after the administration of test substances by using Eddy's hot plate method. The $\%$ analgesic activity (PAA) was calculated by the following formula PAA $=(T-C) / C \times 100, C$ is the reaction time of the control and $T$ is the reaction time of the test compound.

\section{Anti-inflammatory Activity (carrageenan-induced rat paw edema)}

The anti-inflammatory activity was carried out by carrageenan-induced paw edema test. ${ }^{38}$ Wistar albino rats of either sex thirty-three animals (122-140 g.) were divided into eleven groups, each with three animals. The animals of group-I considered as control, were administered with normal saline. The animals of group-II are treated with standard drug indomethacin $(20 \mathrm{mg} / \mathrm{kg})$. Animals of Group-III-XI were administered with the test sample. Test compounds and standard drug indomethacin $(10 \mathrm{mg} / \mathrm{kg}$ ) were suspended in $0.5 \% \mathrm{w} / \mathrm{v}$ of sodium carboxyl methylcellulose (CMC), which was used as a vehicle for the control group.

The rats were dosed with test drugs orally, including the reference standard with the help of an oral feeding needle. After 30 minutes of drug administration, $0.1 \mathrm{~mL}$ of $1 \% \mathrm{w} / \mathrm{v}$ carrageenan solution in saline was injected in the sub plantar region of the left hind paw of control as well as standard and test groups. The increased volume of paw edema (in $\mathrm{mL}$ ) was determined immediately after the injection of carrageenan and 30,60 \& 120 minutes later. The difference between 30 and 60 minutes was taken as edema volume. The $\%$ inhibition of anti-inflammatory activity (PAA) was calculated by the following formula PAA $=(C-T) / C \times 100, C$ is the reaction time of the control and $\mathrm{T}$ is the reaction time of the test compound. Data were expressed as means \pm SEM.

\section{Acknowledgments}

The authors are grateful to the Department of Chemistry, Bapatla Engineering College for providing workspace and are indebted to Bapatla College of Pharmacy for their extended help in providing animals and activity studies. The authors would also like to extend their gratitude to Dr. P. Ashamadhavi, HOD; Sri N. Sudheer, Asst. Professor, Dept. of English for grammatical corrections of the manuscript. 


\section{Supplementary Material}

The NMR spectra of some synthetic compounds, and tabulated details of the analgesic and anti-inflammatory testing data, are given in the Supplementary file linked to this article.

\section{References}

1. Kleeman, A.; Engel, J.; Kutscher, B.; Reichert, D. Pharmaceutical Substances, 3rd Ed.; George Thieme: Stuttgart, New York, NY, 1999, 1190.

2. Theodoridis, G.; In Modern Crop Protection Compounds; Kramer, W.; Schirmer, U., Eds.; Wiley-VCH: Weinheim, 2007, 153.

3. Vicentini, C. B.; Romagnoli, C.; Andreotti, E.; Mares, D. J. Agr. Food Chem. 2007, 55, 10331.

4. Eicher, T.; Hauptmann, S., Edition IInd, 'The Chemistry of Heterocycles: Structure, Reactions, Syntheses, and Applications', 2003, Wiley-VCH, ISBN 3527307206.

5. Krygowski, T. M.; Anulewicz, R.; Cyrafiski, M. K.; Puchala, A.; Rasata, D. Tetrahedron 1998, $54,12295$.

6. Knorr, L. Ber. Deut. Chem. Ges. 1883, 16, 2597.

7. Polshettiwar, V.; Varma, R. S. Tetrahedron Lett. 2008, 49, 397.

8. Arrieta, A.; Carrillo, J. R.; Cossiò, F. P.; Ortiz, A. D.; Gomez-Escalonilla, M.J. ; de la Hoz, A. ; Langa, F . ; Moreno, A. Tetrahedron 1998, 54, 13167.

9. Remy, R. J.; Secrist III, J. A. Nucleosides Nucleotides 1985, 4, 411.

10. Sachchar S. P.; Singh A. K. Int. J. Comp. Socio. 1985, 12, 142.

11. Nada, M. A.; Hamdi, M. H.; Ahmed, S. M.; Omar, A. M. J. Bio. Chem. 2009, 20, 975.

12. Pattan, S. R. Ind. J. Chem. 2009, 48, 1453.

13. Mathew, B.; Suresh, J.; Anbazhagan, S. Amer. J. Chem. Soc. 2012, 2(3), 1.

14. Kumar, A. S.; Ilango, K.; Manikandan, R. S.; Ramalakshmi, N. Eur. J. Chem. 2009, 6, 123.

15. Sahu, S. K.; Banerjee, M.; Samantray, A.; Behera, C.; Azam, M. A. Tropical J. Pharm. Res. 2008, 7, 961.

16. Panneer, S. T.; Saravanan, G.; Prakash, C. P.; Kumar, P. D. African J. Chem. 2011, 1, 126.

17. Mohareb, R. M.; El-Sayed, N. N.; Abdelaziz, M. A. Molecules 2012, 17, 8449.

18. El-Sayed, W. A.; Flefel, E. M.; Morsy, M. H. Der Pharm. Chem. 2012, 4, 23.

19. Bole, S. B.; Nargund, L. V.; Nargund, R.; Devaraju, K. S.; Vedamurthy, A. Int. J. Pharm. Sci. 2011, 3, 161.

20. Tarun, S.; Mithilesh, Raju, S.; Pooja, C.; Saraf, S. K. Int. J. Res. Pharm. Sci. 2012, 2, 81.

21. Olofson, R. A.; and Kendall, R. V. J. J. Org. Chem. 1970, 35, 2246.

22. Lidström, P.; Tierney, J.; Wathey B.; and Westman, J. Tetrahedron 2001, 57, 9225.

23. Synthetic Applications of 1,3-Dipolar Cycloaddition Chemistry: toward Heterocycles and Natural Products,

(Eds. Padwa, A.; Pearson W. H.) Wiley, Hoboken, 2003, 681.

24. Fustero, S.; Sanchez-Rosell, M.; Barrio, P.; Simn-Fuentes, A. Chem. Rev. 2011, 111, 6984

25. Babinski, D. J.; Aguilar, H. R.; Still, R.; Frantz, D. E. J. Org. Chem. 2011, 76, 5915.

26. Simovic, D. M. D., Marks, V.; Chatfield, D. C.; Rein, K. S. J. Org. Chem. 2006, 71, 650.

27. Kumar, R.; Verma, D.; Mobin, S. M.; Namboothiri, I. N. N. Org. Lett. 2012, 14, 4070.

28. Foster, R. S.; Jakobi H.; Harrity, J. P. A. Org. Lett. 2012, 14, 4858.

29. Gioiello, A.; Khamidullina, A.; Fulco, M. C.; Venturoni, F.; Zlotsky, S.; Pellicciari, R. Tetrahedron Lett. 2009, $50,5978$. 
30. Kumar, R.; Namboothiri, I. N. N. Org. Lett. 2011, 13, 4016.

31. Ichikawa, H.; Watanabe, R.; Fujino, Y.; Usami, Y. Tetrahedron Lett. 2011, 52, 4448.

32. Browne, D. L.; Vivat, J. F.; Plant, A.; Gomez Bengoa, E.; Harrity, J. P. A. J. Am. Chem. Soc. 2009, 131, 7762.

33. Furniss, B. S.; Hannaford, A. J.; Rogers, V.; Smith, P. W. G.; Tatchell, A. R. in Vogel's Textbook of Practical Organic Chemistry, ELBS: UK, 4th edn, 1978, 802.

34. Madhava Rao, V.; Sudhakar, D.; Siddhaiah, V.; Venkatarao, C. Int. J. Synth. Characterisation 2008, 1, 17. 35. Priyadarsini, P.; Ujwalaa, B.; VenkataRao, C.; MadhavaRao, V. Der Pharmacia Lettre 2012, 4, 1123.

36. Pinto, D. C.G.A. ; Silva, A.M.S. ; Cavaleiro, J.A.S. Tetrahedran 1999, 55, 10187.

37. Verma, A. K.; Martin, A. Singh A. K. Asian J. Biomed. Pharm. Sci. 2014, 4 (37), 21.

38. Dhingra, A. K.; Chopra, B.; Dass, R.; and Mittal, S .K. Int. J. Med. Chem. 2016, 1. 